\title{
HyperAdapt: Enabling Aspects for XML
}

\author{
Matthias Niederhausen ${ }^{1}$, Sven Karol ${ }^{2}$, Uwe Aßmann ${ }^{2}$, and Klaus Meißner ${ }^{1}$ \\ 1 Technische Universität Dresden \\ Chair of Multimedia Technology \\ 01062 Dresden, Germany \\ \{matthias.niederhausen, kmeiss\}@inf .tu-dresden.de \\ 2 Technische Universität Dresden \\ Software Technology Group \\ 01062 Dresden, Germany \\ \{sven.karol, uwe.assmann\}@tu-dresden.de
}

\begin{abstract}
Aspect orientation offers an intuitive way to specifiy adaptivity for web applications, but despite its advantages, the approach still lacks acceptance. We argue that there are two main reasons for this: First, aspects make implicit assumptions on the underlying system and can thus produce invalid behavior if that system is changed. Second, the lack of concepts for dealing with aspect interactions places a heavy burden on the use of multiple aspects. In this paper we discuss how HyperAdapt addresses these problems, paving the road towards a productive use of aspect orientation in the domain of web applications.
\end{abstract}

\section{Introduction}

Developing adaptive hypermedia applications is a very challenging and demanding task. In order to achieve a single adaptation goal (e.g., adapting to a special device), many different application parts have to be edited in a consistent way. This process gets even more complicated if there are multiple different adaptation goals. To deal with this complexity, we apply aspect-oriented programming (AOP) to place adaptivity into separate modules - so-called adaptation aspects. The growing number of approaches that leverage aspect orientation to model adaptivity [1|2:3 clearly demonstrates the attractiveness of this solution. In contrast to these existing solutions, we apply aspects not on a model level, but on XML. However, aspect orientation in this context still has several open issues:

1. Untyped Aspect Weaving. In hypermedia applications, aspects are usually woven into XML documents. To guarantee validity of the resulting document, weaving operations must support typed weaving.

2. Interaction of Aspects. Aspects may conflict if they address the same part of a document. Since it is hard to keep an overview of such conflicts, it is imperative to provide means for detecting and potentially resolving them.

3. Constrained Aspects. If a document contains protected parts that should not be adapted, it can be helpful to impose further restrictions on documents or aspects beyond type-safety. Thus, techniques are needed to specify constraints for documents and aspects. 


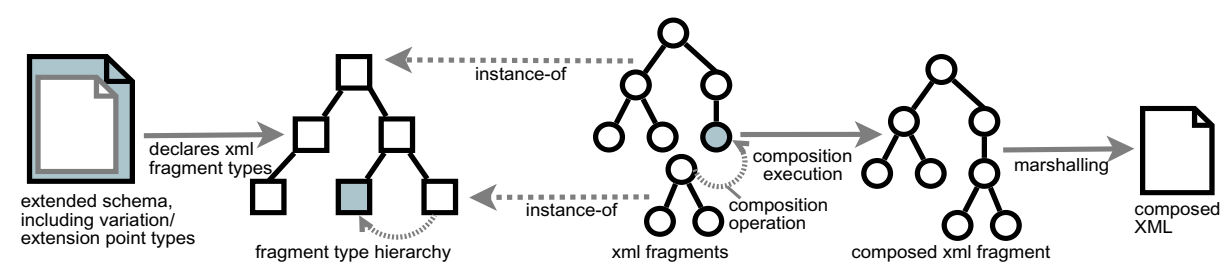

Fig. 1. Invasive composition of XML documents

In the remainder of this paper we explain how HyperAdapt tackles the above issues. Sect. 2 introduces a type-safe aspect weaving approach. Sect. 3 investigates how to detect aspect interactions, while Sect. 4 discusses aspect conflict resolution and constraint-based weaving. Finally, Sect. 5 concludes the paper.

\section{Safe Weaving of Web Adaptation Aspects}

An appropriate composition technique for safe aspect weaving has to consider the specific properties of XML. At runtime, XML documents are usually represented as trees typed by XML schemas, which are comparatively similar to a contextfree grammar describing the abstract syntax of a language.

Invasive Software Composition (ISC) 4] is a powerful technique to create composition systems for tree-shaped components, called fragments in this context. It has proven to be expressive enough to recreate several other component-based concepts and ensures syntactic type-safe composition w.r.t. the component language. ISC has made its way to several orthogonal domains. For instance, the COMPOST 11 system recreates aspect orientation and generics for Java 1.4 . Also, Modular XCerpt provides the XCerpt query language with a module system [5]. To reduce implementation effort, recent research developed grammar-based ISC [6], which allows to declaratively specify composition systems based on markup and extension of context-free grammars. However, the approach has not yet been applied to XML languages. Hence, to be applicable to XML documents, we attune the approach in two ways:

First, we extend grammar-based ISC to support XML, such that every composition step composes fragments according to a given XML schema (see Figure 1). Furthermore, we develop an approach to declare extension and variation points (see filled symbols in Figure 1) in XML documents as well as complex composition operators and evaluate it by means of prototype composition systems. Second, we use a weaving pipeline for composing aspects of general XML-based languages. Each stage in this pipeline provides output parameters and requires input parameters which can be typed according to a given schema. This allows for modular type-safe definitions of composition steps even for different XML dialects. Moreover, it provides a grounding for conflict resolution techniques and constraints imposed on aspects as discussed in Sect. 4.

${ }_{1}^{1}$ The compost system. http://www.the-compost-system.org 


\section{Investigation of Aspect Interactions}

Typically, aspects resemble adaptation techniques, as identified by the adaptive hypermedia community (e.g., [78), and are applied to components in the web application. Dependant on context parameters and runtime state of the web application, aspects usually crosscut several XML documents. Thus, they are quite similar to aspects in aspect-oriented programming 9], which are applied to a pointcut. However, in contrast to general AOP, a clear separation between static (e.g., some node in the document) and dynamic parts (e.g., the context) of a pointcut exists. We argue that these and other special properties of adaptation aspects can be used to better analyse aspect interactions. To this end, we classify frequently used adaptation techniques by their potential for conflicts. For example, an advice removing a fragment can have an impact on other advices with overlapping pointcuts (subsequent advices cannot match the removed fragment anymore), but this may also be desired by the author. In contrast, an advice replacing all text by a localised version and another one replacing certain images by text have a closer interdependency, as the first should be executed only after the second has taken effect.

In a next step, we plan to extend well-known adaptation techniques by a description that offers semantic information on the technique. Starting from the classification and the semantic description, we can then analyse adaptation techniques and aspects to identify potential conflicts. There are multiple options for doing so: First, conflicts are often found when two advices address the same component, therefore we intend to check on these cases first. Second, we plan to employ a conflict matrix that marks often-found interactions, such as the example given above. Third, we can apply confluence tests to identify whether the order of advices has an influence on the resulting document: if this is not the case, we can be sure that there are no conflicts. And fourth, we can combine static analysis based on type information derived from XML schema and analysis of the semantic description to find conflicts before aspects are deployed.

\section{Constraint-Based Adaptation}

As discussed in Sect. 3, aspects cannot always be completely orthogonal. Often enough, this is even desired by the author (e.g., internationalising a text and at the same time removing it for unauthorised users). A correct resolution has to bring the involved aspects into the right order. One way to do so is to extend the conflict matrix and supply a proposed weaving order for two given aspects. Another option is to define precedence for aspects, depending on the narrowness of their pointcut. For example, if the author has defined a separate adaptation for one particular document fragment, this should always have precedence over a document-wide adaptation that also includes this joinpoint.

Beyond constraints defined by the aspect author, XML document authors may impose constraints on parts of their document. For example, the author may restrict the replacement of images at certain positions or he may require 
inserted fragments to adhere to a certain structure (e.g., an altering list of text and images). To provide a framework for definition of such constraints, we borrow concepts from contract-based programming [10]. Preconditions may contain structural information on documents before an aspect is applied, postconditions then describe the resulting structure. Thus, contracts can be used to narrow the application of aspects to certain parts of a document.

\section{Summary}

In this work, we introduced the problem of aspect interactions in the domain of XML documents. We further proposed strategies for dealing with such interactions (e.g., confluence check) and an extension to AOP such that sophisticated contracts between aspects and the application can be defined. With the help of the suggested approach, aspect orientation unlocks its full potential for documents, allowing to cleanly separate adaptation from the rest of a web application.

As the next steps, we extend grammar based Invasive Software Composition to support XML languages and create a prototype weaving pipeline. Additionally, we will investigate adaptation techniques w.r.t. their conflict potential. These initial steps will then give us important feedback on our design of a contract model for aspects.

\section{References}

1. Baumeister, H., Knapp, A., Koch, N., Zhang, G.: Modelling adaptivity with aspects. In: Lowe, D.G., Gaedke, M. (eds.) ICWE 2005. LNCS, vol. 3579, pp. 406-416. Springer, Heidelberg (2005)

2. Schauerhuber, A., Wimmer, M., Schwinger, W., Kapsammer, E., Retschitzegger, W.: Aspect-oriented modeling of ubiquitous web applications: The aspectwebml approach. In: ECBS MBD 2007, Tucson, Arizona (2007)

3. Casteleyn, S., Van Woensel, W., Houben, G.-J.: A semantics-based aspect-oriented approach to adaptation in web engineering. In: HT 2007: Proceedings of the eighteenth conference on Hypertext and hypermedia, pp. 189-198. ACM, New York (2007)

4. Aßmann, U.: Invasive Software Composition, 1st edn. Springer, Heidelberg (2003)

5. Aßmann, U., Berger, S., Bry, F., Furche, T., Henriksson, J., Johannes, J.: Modular web Queries-From rules to stores (2007)

6. Henriksson, J.: A Lightweight Framework for Universal Fragment Composition. Ph.D thesis, Technischen Universität Dresden (2008)

7. Bunt, A., Carenini, G., Conati, C.: Adaptive content presentation for the web. In: Brusilovsky, P., Kobsa, A., Nejdl, W. (eds.) Adaptive Web 2007. LNCS, vol. 4321, pp. 409-432. Springer, Heidelberg (2007)

8. Brusilovsky, P.: Adaptive navigation support. In: Brusilovsky, P., Kobsa, A., Nejdl, W. (eds.) Adaptive Web 2007. LNCS, vol. 4321, pp. 263-290. Springer, Heidelberg (2007)

9. Kiczales, G., Mendhekar, A., Lamping, J., Maeda, C., Lopes, C.V., Loingtier, J.M., Irwin, J.: Aspect-Oriented programming. In: Aksit, M., Matsuoka, S. (eds.) ECOOP 1997. LNCS, vol. 1241, pp. 220-242. Springer, Heidelberg (1997)

10. Meyer, B.: Applying 'design by contract'. Computer 25(10), 40-51 (1992) 